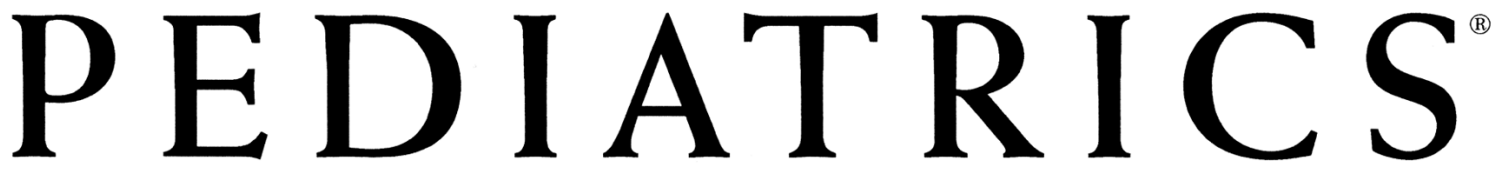

OFFICIAL JOURNAL OF THE AMERICAN ACADEMY OF PEDIATRICS

\title{
A Survey of the Use of Off-Label and Unlicensed Drugs in a Dutch Children's Hospital
}

Geert W. 't Jong, Arnold G. Vulto, Matthijs de Hoog, Kirsten J. M. Schimmel, Dick Tibboel and John N. van den Anker

Pediatrics 2001;108;1089-1093

DOI: $10.1542 /$ peds.108.5.1089

This information is current as of November 20, 2006

The online version of this article, along with updated information and services, is located on the World Wide Web at:

http://www.pediatrics.org/cgi/content/full/108/5/1089

PEDIATRICS is the official journal of the American Academy of Pediatrics. A monthly publication, it has been published continuously since 1948. PEDIATRICS is owned, published, and trademarked by the American Academy of Pediatrics, 141 Northwest Point Boulevard, Elk Grove Village, Illinois, 60007. Copyright $\odot 2001$ by the American Academy of Pediatrics. All rights reserved. Print ISSN: 0031-4005. Online ISSN: 1098-4275.

\section{American Academy of Pediatrics}




\title{
A Survey of the Use of Off-Label and Unlicensed Drugs in a Dutch Children's Hospital
}

\author{
Geert W. 't Jong, MSc*; Arnold G. Vulto, PharmD\&; Matthijs de Hoog, MD*;

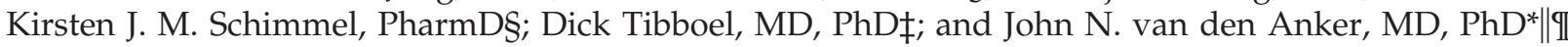

\begin{abstract}
Background. The treatment of pediatric patients with drugs in hospitals is being impeded by a shortage in the availability of licensed drugs in an appropriate formulation. We have studied the extent of use of drugs that are not licensed for use in children (unlicensed) and drugs that are used outside the terms of the product license (off-label). We conducted this study in a Dutch academic children's hospital.

Methods. In a prospective study of 5 weeks' duration, we reviewed drug prescriptions in a pediatric ward and 3 intensive care units. We classified the prescribed drugs in 3 main categories-licensed, unlicensed, and off-label-and determined the nature of their unlicensed and off-label use.

Results. Two thousand one hundred thirty-nine courses of drugs were administered to 237 patients in 442 patient-days. Of 2139 prescriptions, 725 (34\%) were licensed, $1024(48 \%)$ were unlicensed, and 390 (18\%) were off-label. In $392(90 \%)$ of 435 patient-days, children received 1 or more courses of an unlicensed or off-label drug prescription in hospital.

Conclusion. With regard to the availability of drugs of proven quality and adequate license for pediatric patients in hospital, dramatic shortcomings exist. As a result, drug legislation originally designed to protect patients and prescribing physicians against unsafe drug use and unjustified claims has turned into an insurmountable threshold to make proper drugs available for a vulnerable minority of patients. Pediatrics 2001;108:10891093; children, drug use, labeling, unlicensed, off-label, licensing, drugs.
\end{abstract}

ABBREVIATIONS. MCU, medium care unit; NICU, neonatal intensive care unit; SICU, surgical intensive care unit; PICU, pediatric intensive care unit.

$\mathrm{M}$ any commercially available drugs are only licensed for use in adults and are not used according to the product licensing in pediatric practice. Furthermore, for many drugs, the available formulations are unsuitable for pediatric

From the Departments of *Pediatrics and $\ddagger$ Pediatric Surgery, and §Hospital Pharmacy, Sophia Children's Hospital, Erasmus Medical Center Rotterdam (EMCR); Rotterdam, The Netherlands; ||Division of Pediatric Clinical Pharmacology and Medical Toxicology, Columbus Children's Hospital, Columbus, Ohio; and IIDepartment of Pediatrics, Ohio State University, Columbus, Ohio.

Received for publication Sep 28, 2000; accepted Jun 13, 2001.

Reprint requests to (J.N.vdA.) Division of Clinical Pharmacology and Toxicology, Children's Hospital, Columbus, OH 43205. E-mail: vandenankerj@ pediatrics.ohio-state.edu

PEDIATRICS (ISSN 0031 4005). Copyright @ 2001 by the American Academy of Pediatrics. use, $^{1}$ and for many compounds in common use in pediatrics, preparations are not commercially available at all. There are several reasons for this situation. According to the modern standards of drug evaluation, obtaining a product license for a specific drug for a specific indication in a specific patient group necessitates extensive research. With children forming only a minority in the drug market, the profitdriven drug industry by nature is reluctant to invest in pediatric drug studies. In addition, fear is growing for unforeseen and hard-to-study long-term side effects. As a result, drug legislation originally designed to protect patients and prescribing physicians against unsafe drug use and unjustified claims has turned into a barrier to making proper drugs available for a vulnerable minority of patients.

Other reasons are the ethical problem of research in children, the reluctance of parents to allow their children to participate in drug trials, and the technical challenges small study participants bring along. Possibly because of the underestimation of the problem, there is lack of funding from government, health care providers, and industry. As a result, pediatric drug trials are relatively scarce and in many cases, contain only a limited number of patients.

Consequently, most drugs used in clinical practice in pediatrics are not licensed for children, ${ }^{2-6}$ and this has lead to children being referred to as "therapeutic orphans." ${ }^{7}$ Use of these drugs is sometimes based on the modification of adult formulations and dosage strengths and extrapolation of doses used in adults. This neglects the important differences between adults and children in development and drug metabolism and excretion. ${ }^{8}$ Often, dosage regimens are based on clinical trials and published experience in children, although not submitted to licensing scrutiny.

Surveys in the United Kingdom by Turner et al, and by others, ${ }^{9-15}$ have shown that many drugs prescribed to children in pediatric, and especially neonatal, care are not licensed for children, or are prescribed "off-label" (ie, outside the terms of the product license). With tightening rules in medical practice and an increasing number of lawsuits, pediatricians are in an unenviable position. In the United States, about $80 \%$ of all drugs approved for the market lack partial or complete information in the label pertaining to use in pediatric patients. ${ }^{16-18}$ The US Food and Drug Administration has implemented new regulations to increase the number of drugs 
available for pediatric use. ${ }^{19,20}$ In Europe, similar changes are under discussion, currently only with very limited success. ${ }^{21,22}$

In contrast to many other European countries, most Dutch hospital pharmacies provide their pediatric wards with fully "homemade" pediatric formulations or modified commercial preparations (eg, strength-adapted suspensions, capsules) on a large scale. We wanted to investigate the licensing status of the drugs commonly used in a pediatric academic setting against this background. We therefore studied in detail all drugs prescribed in 4 hospital units in our academic children's hospital.

\section{METHODS}

\section{Setting}

Data were retrieved from 4 hospital units of the Sophia Children's Hospital in Rotterdam, The Netherlands, an academic children's hospital. This highly specialized hospital provides the Rotterdam region with care for children that are seriously ill, and in need of specific care. During a 5-week period (February to March 1999), we prospectively investigated 1 large, medium-care unit and 3 intensive care units. These 4 hospital units were as follows: medium care unit (MCU), 56 beds/cribs; neonatal intensive care unit (NICU), 28 cribs; surgical intensive care unit (SICU), 18 beds / cribs; and pediatric intensive care unit (PICU), 14 beds/cribs.

\section{Design}

To determine the drug licensing status of drugs prescribed to children in this hospital, we prospectively gathered prescription data study in a dynamic cohort. We studied all patients that were hospitalized in 1 of the hospital units during the study period. We defined each event in which the prescriptions of an individual patient on a separate day have been investigated, as 1 "patientday." Each hospital unit was studied for 1 day each week for 5 consecutive weeks, and was visited on a different day each week.

Data collection included: unit involved, week number, date of birth, age, weight, gender, diagnosis or reason of admission, drugs administered, form and route of administration, dose, frequency, and indication for use. The use of the following drugs was not recorded: standard intravenous crystalloid fluids, blood products, total parental nutrition, and oxygen therapy.

\section{Classification}

All drugs administered were assessed for licensing status by way of a classification system specially adapted to the Dutch situation, although primarily based on a classification system described and used in previous published studies in the United Kingdom. ${ }^{10}$

In the main classification category, 4 main groups of prescriptions were defined: 1) proprietary medications; 2) generic, or nonproprietary medication; 3 ) commercial formulations modified by the hospital pharmacy (modified); and 4) medications manufactured by the hospital pharmacy (home label).

A prescription was automatically defined unlicensed if "modified" or "home label" was applicable. In case of a modification, the commercial manufacturer would not be liable for the altered administered prescription, because the license of the original product is not applicable to the modified product. In case of a home-label drug, no license was applicable, because it was produced by the hospital pharmacy itself. These prescriptions were not further classified for off-label use because the lack of proper information texts.

If a prescription was a propriety or generic product, we further classified for 5 other classification categories, namely age, dosage form and route of administration, daily dosage used, number of doses per day, and indication. If the prescription in 1 or more of the other classification categories was not according to registration, it was defined "off-label." Exceptions were made when the prescription was "not licensed for use in children" or "contraindicated for use in children" (contraindicated), or when doses for use in children were not mentioned in the reference (no information on use in children), in which case the prescription was defined "unlicensed."

The primary reference source used was the Repertorium 98/9923 (an official Dutch compendium with approved drug information on drug specialties). The alternative source of information was the Farmacotherapeutisch Kompas $98^{24}$ (a compendium provided by the Dutch National Health Service), which was used if the drug involved was not available as a proprietary medication, and therefore not mentioned in the Repertorium 98/99.

\section{RESULTS}

During the 5-week study period in February and March 1999, 237 patients were included in the study in a total of 448 patient-days. The ages of the patients at admission ranged between 0 days and 17 years. Of 237 patients, $129(54 \%)$ were male. The 4 hospital units admitted 110 (MCU), 64 (NICU), 33 (SICU), and 31 (PICU) patients, receiving 905, 621, 308, and 305 prescriptions, respectively (Table 1 ). Fourteen patients switched from an intensive care unit to the MCU during hospitalization. Most important reasons for admission were: prematurity, bronchopulmonary dysplasia, cardiac malformations, oncology, cystic fibrosis, chronic renal failure, and asthmatics.

In $1414(66 \%)$ of the 2139 prescriptions, drugs were either unlicensed $(1024 ; 48 \%)$ or off-label $(390$; $18 \%)$. Three hundred ninety-two (90\%) of all 435 patient-days contained unlicensed drugs or off-label prescription. Of all prescriptions, 193 (9\%) were commercial formulations modified by the hospital pharmacy (modified), and $567(27 \%)$ were medications manufactured by the hospital pharmacy (home label). This percentage was even higher in NICU. Three hundred thirty-four $(16 \%)$ drug prescriptions were off-label for dose, and $100(5 \%)$ were off-label for age (Table 2).

A total of 189 drugs were prescribed, the most frequently encountered drugs in the study were nys-

TABLE 1. Patient Characteristics

\begin{tabular}{|c|c|c|c|c|c|}
\hline $\begin{array}{c}\text { Hospital Unit } \\
\text { (Number of Beds/Cribs) }\end{array}$ & $\begin{array}{l}\mathrm{MCU} \\
(56)\end{array}$ & $\begin{array}{l}\text { NICU } \\
(28)\end{array}$ & $\begin{array}{l}\text { SICU } \\
(18)\end{array}$ & $\begin{array}{l}\text { PICU } \\
(14)\end{array}$ & $\begin{array}{l}\text { Total } \\
(116)\end{array}$ \\
\hline Patients admitted & 110 & 66 & 34 & 27 & 237 \\
\hline \multicolumn{6}{|l|}{ Gender } \\
\hline Male & 64 & 33 & 14 & 18 & 129 \\
\hline Female & 46 & 33 & 20 & 9 & 108 \\
\hline \multicolumn{6}{|l|}{ Age } \\
\hline Age range & $4 \mathrm{~d}-17 \mathrm{y}$ & $0 \mathrm{~d}-6 \mathrm{mo}$ & $6 \mathrm{~d}-16 \mathrm{y}$ & $4 d-15 y$ & $0 \mathrm{~d}-17 \mathrm{y}$ \\
\hline Median age & $4.5 \mathrm{y}$ & $12 \mathrm{~d}$ & $54 \mathrm{~d}$ & $2.2 \mathrm{y}$ & $8.5 \mathrm{mo}$ \\
\hline Patient-days & $186^{\circ}$ & 130 & 70 & 49 & 435 \\
\hline Prescriptions & 905 & 621 & 308 & 305 & 2139 \\
\hline Prescriptions per patient-day & 4.9 & 4.8 & 4.4 & 6.2 & 4.9 \\
\hline
\end{tabular}


TABLE 2. Drug Classification Subgroups

\begin{tabular}{|c|c|c|c|c|c|c|}
\hline Category & Subcategory & $\begin{array}{c}\text { MCU } \\
(n=905)\end{array}$ & $\begin{array}{c}\text { NICU } \\
(n=621)\end{array}$ & $\begin{array}{c}\text { SICU } \\
(n=305)\end{array}$ & $\begin{array}{c}\text { PICU } \\
(n=308)\end{array}$ & $\begin{array}{c}\text { Total } \\
(n=2139)\end{array}$ \\
\hline \multirow{4}{*}{$\begin{array}{l}\text { Licensed } \\
\text { Unlicensed }\end{array}$} & & $375(41 \%)$ & $153(24 \%)$ & $100(32 \%)$ & $97(32 \%)$ & $725(34 \%)$ \\
\hline & Contraindicated & $9(1 \%)$ & $5(1 \%)$ & $7(2 \%)$ & $1(<1 \%)$ & $22(1 \%)$ \\
\hline & No information on use in children & $112(12 \%)$ & $46(7 \%)$ & $33(11 \%)$ & $51(17 \%)$ & $242(11 \%)$ \\
\hline & $\begin{array}{l}\text { Prescriptions manufactured or modified } \\
\text { by the hospital pharmacy }\end{array}$ & $239(26 \%)$ & $333(54 \%)$ & $80(26 \%)$ & $108(35 \%)$ & $760(36 \%)$ \\
\hline Total unlicensed & & $360(40 \%)$ & $384(62 \%)$ & $120(39 \%)$ & $160(52 \%)$ & $1024(48 \%)$ \\
\hline \multirow[t]{11}{*}{ Off-label } & Dose & $53(6 \%)$ & $11(2 \%)$ & $21(7 \%)$ & $17(6 \%)$ & $102(5 \%)$ \\
\hline & Dose and frequency & $50(6 \%)$ & $7(1 \%)$ & $8(3 \%)$ & $20(7 \%)$ & $85(4 \%)$ \\
\hline & Dose and indication & $15(2 \%)$ & & $1(<1 \%)$ & $1(<1 \%)$ & $17(1 \%)$ \\
\hline & Dose, frequency, and indication & $4(<1 \%)$ & $3(<1 \%)$ & $13(4 \%)$ & & $20(1 \%)$ \\
\hline & Dose, frequency, and route & $2(<1 \%)$ & $14(2 \%)$ & & $1(<1 \%)$ & $17(1 \%)$ \\
\hline & Dose, frequency, and age & $33(4 \%)$ & $19(3 \%)$ & $37(12 \%)$ & $1(<1 \%)$ & $90(4 \%)$ \\
\hline & Dose, frequency, age, and route & $1(<1 \%)$ & & & $2(1 \%)$ & $3(<1 \%)$ \\
\hline & Age & $4(<1 \%)$ & & $1(<1 \%)$ & $2(1 \%)$ & $7(<1 \%)$ \\
\hline & Frequency & $4(<1 \%)$ & $29(5 \%)$ & $6(2 \%)$ & $2(1 \%)$ & $41(2 \%)$ \\
\hline & Indication & $2(<1 \%)$ & $1(<1 \%)$ & $1(<1 \%)$ & $2(1 \%)$ & $6(<1 \%)$ \\
\hline & Route of administration & $2(<1 \%)$ & & & & $2(<1 \%)$ \\
\hline Total off-label & & $170(19 \%)$ & $84(14 \%)$ & $88(29 \%)$ & $48(16 \%)$ & $390(18 \%)$ \\
\hline
\end{tabular}

tatin, cisapride, and acetaminophen (Table 3). The most frequently encountered unlicensed drugs and off-label prescriptions in use were cisapride, caffeine, and tobramycin (Table 4). Off-label use is mainly attributable to use of different dose and frequency to that recommended in the product license. Ipratropium, budesonide, and salbutamol (Atrovent, Pulmicort, and Ventolin) are used in various combinations and dosage proportions. The components are dissolved in $\mathrm{NaCl} 0.9 \%$ by the hospital pharmacy, and then used in an aerosol.

\section{DISCUSSION}

The licensing status of many of the drugs commonly used in our academic children's hospital is inadequate. Results show a high prevalence of unlicensed $(44 \%)$ and off-label $(15 \%)$ drug use in our MCU. We found even larger proportions of unlicensed and off-label drug use in intensive care, and especially in the NICU, as expected. Lack of flexible pediatric formulations and lack of drugs properly licensed for newborns and infants are important cofactors. We found a strikingly high use of home-label prescriptions in NICU (41\%), which is mainly caused by the lack of flexible pediatric formulations, which the hospital pharmacy tries to overcome by manufacturing the needed formulations themselves.

We expected the number of drugs used (4.9 prescriptions per patient day) in the MCU to be lower than in the ICUs. However, no significant difference is found. This is probably attributable to the relatively high prevalence of chronic respiratory illness (cystic fibrosis, asthma) in this patient population.
Studies in the United Kingdom by Choonara et $\mathrm{al}^{11-14}$ showed similar results concerning the licensing status of prescribed drugs. When comparing our study to the UK studies, several aspects in design have to be considered. The extensive use of medications modified by the hospital pharmacy (modified) and formulations manufactured by the hospital pharmacy (home label) in the Netherlands compared with the studies performed in the United Kingdom results in very high percentages of unlicensed drug prescription. The difference between these studies is attributable to the pharmacy strategy to dedicate resources to clinical pharmacy service provision rather than manufacturing, which is followed by most departments in the United Kingdom. UK pharmacists are allowed to extemporaneously dispense any drug for an individual patient, but good manufacturing practice regulations must rightly be followed if done on a larger scale. In the Netherlands, hospitals pharmacies often manufacture on a large scale for cost-saving reasons. Besides that, the number of drugs licensed for use in the Netherlands is smaller, also resulting in a higher proportion of modified medications and home label formulations; they together make up for $36 \%$ of all prescriptions. The method of data collection differed not only in length of study period, but also in data collection interval. In an international study in pediatric wards that we participated in, ${ }^{25}$ we used some of the data from this study. Preliminary results of this study have been reported in abbreviated form. ${ }^{26}$ The classification system used in these preliminary results was slightly different, but to facilitate comparison we adapted

TABLE 3. The 5 Most Frequently Prescribed Drugs in 4 Different Hospital Units During the 5-Week Investigation Period

\begin{tabular}{|c|c|c|c|c|c|c|c|c|c|}
\hline $\mathrm{MCU}$ & $\% *$ & NICU & $\%$ & SICU & $\%$ & PICU & $\%$ & Total & $\%$ \\
\hline Acetaminophen & 4 & Caffeine & 11 & Nystatin & 17 & Amphotericin B & 9 & Nystatin & 5 \\
\hline Amphotericin B & 3 & Nystatin & 9 & Cisapride & 8 & Cisapride & 7 & Cisapride & 4 \\
\hline Ondansetron & 3 & Vitamin $\mathrm{D}_{3}$ and $\mathrm{E}$ & 7 & Acetaminophen & 5 & Acetaminophen & 6 & Acetaminophen & 4 \\
\hline Furosemide & 2 & Ipratropium and salbutamol & 5 & Cefotaxime & 5 & Cotrimoxazole & 6 & Caffeine & 4 \\
\hline Spironolactone & 2 & Tobramycin & 5 & Furosemide & 4 & Furosemide & 6 & Furosemide & 3 \\
\hline
\end{tabular}

*\% indicates the percentage of all drug prescriptions in the hospital unit. 
TABLE 4. The 5 Most Frequently Used Unlicensed and Off-Label Drug Prescriptions in 4 Different Hospital Units During the 5-Week Investigation Period

\begin{tabular}{|c|c|c|c|c|}
\hline MCU & NICU & SICU & PICU & Total \\
\hline Pancreatin & Caffeine & Cisapride & Cisapride & Cisapride \\
\hline Spironolactone & Vitamin $\mathrm{D}_{3}$ and $\mathrm{E}$ & Gaviscon & Spironolactone & Caffeine \\
\hline Furosemide & Ipratropium and salbutamol & Acetaminophen & Ranitidine & Tobramycin \\
\hline Tobramycin & Tobramycin & Morphine & Furosemide & Spironolactone \\
\hline Phytomenadione & Dexamethasone & Phytomenadione & Trimeprazine & Furosemide \\
\hline
\end{tabular}

our data to the classification system used in the surveys by Turner et al. ${ }^{10}$

It is important to recognize that off-label or unlicensed use of a drug may not be an inappropriate use (because of reasonable research based foundations of prescription protocol), but may be judged as such when the legal liability of the physician would be questioned in court, because the prescription is not in accordance with labeling information. In our pediatric wards, most drug use is based either on longstanding experience or evidence obtained from the literature. The majority of drugs are prescribed within established protocols. However, the same standards of efficacy and safety cannot be applied as for the adult population. Drugs that were prescribed while contraindicated or unlicensed for age may be particularly unsafe, considering the lack of research completed on these drugs, and the potency of these drugs. They included vigabatrin (unlicensed in children $<10 \mathrm{~kg}$ ) and ciprofloxacin (contraindicated).

Results give a good impression of the extent of unlicensed and off-label drug prescription in very specialized pediatric care in the Netherlands. In general hospital pediatric care, and care provided to children by general practitioners, hardly any information is available on the extent of unlicensed and off-label drug prescription. We hope that future research in these areas will provide us of better knowledge of the extent of this problem.

We strongly support the Food and Drug Administration's Pediatric Rule. ${ }^{19}$ Despite various gaps in these regulations, they are very important for the accomplishment of equality in safety and efficacy of pharmaceutical products in adults and children. ${ }^{27,28}$ The extension of prescribing information of already licensed products with pediatric data are a matter of public interest and therefore should be solved between the industry and the medical community with public support. In return for public support, the license holder should be willing to develop flexible pediatric formulations (like droplets, suspensions, linctus, different strengths, capsules, etc). Older drugs that do not have a patent are however excluded from this rule, despite the fact that they are frequently used. A more difficult problem is to make currently unavailable preparations in need, more generally available. In most countries, according to good manufacturing practice regulations hospital pharmacies are not able to manufacture drugs on a larger scale than facilitation of its hospital(s) requires. It is of crucial importance to investigate whether manufacturers of generic drug products are able to produce certain products on a continent-wide scale (Europe, United States), provided that the in- ternational pediatric community would be able to standardize their practices to make this effort economically reasonable. International collaboration could be realized and coordinated through organizations like the European Network for Drug Investigation in Children, ${ }^{29}$ the American Society for Clinical Pharmacology and Therapeutics, the European Society for Clinical Pharmacology, and the Neonatal and Pediatric Pharmacists Group in the United Kingdom. We hope that drug regulation authorities would support this movement by facilitating an orphan status of such products, thereby reducing the investment risk of the producer. Accumulation of pediatric drug data could be an important strategy, given that efficacy and safety information becomes available beyond reasonable doubt and without very high financial risks for the license holder.

Although drug regulations in general are intended for protection of patients and prescribing physicians, society should be willing to pay the price when side effects of such regulations become counterproductive and unacceptable for children who constitute the future of the society. We have an obligation to investigate seriously every possibility to reverse this highly unfortunate situation in which the progress of medical care is not available for the most vulnerable and defenseless among us.

\section{ACKNOWLEDGMENTS}

The financial support of the Sophia Foundation for Scientific Research through research grant SSWO No. 293 is gratefully acknowledged.

We thank Dr S. N. de Wildt and Dr L. van Rossum for their assistance in methodology, as well as all clinical pediatricians that contributed to information gathering for the study: Dr C. E. de Bel, Dr G. J. Damen, Dr L. Langendonck, Dr G. J. du Marchie Servaas, Dr B. J. Sibbles, and Dr L. van Veen.

\section{REFERENCES}

1. Nahata MC. Lack of pediatric drug formulations. Pediatrics. 1999;104: 607-609

2. Prescribing unlicensed and off-label drugs for unlicensed indications. Drug Ther Bull. 1992;35:97-99

3. Nahata MC. Need for conducting research on medications unlabeled for use in pediatric patients. Ann Pharmacother. 1994;28:1103-1104

4. Gervais A. Comment: medications unlabeled for use in children [letter; comment]. Ann Pharmacother. 1995;29:1052

5. Kauffman RE. Status of drug approval processes and regulation of medications for children [editorial]. Curr Opin Pediatr. 1995;7:195-198

6. American Academy of Pediatrics, Committee on Drugs. Guidelines for the ethical conduct of studies to evaluate drugs in pediatric populations. Pediatrics. 1995;95:286-294

7. Shirkey H. Therapeutic orphans. J Pediatr. 1968;72:119-120

8. Christensen ML, Helms RA, Chesney RW. Is pediatric labeling really necessary? Pediatrics. 1999;104:593-597

9. Nahata MC. Licensing of medicines for children in the USA. Paediatr Perinat Drug Ther. 1997;1:50-51 
10. Turner S, Nunn A, Choonara I. Unlicensed drug use in children in the UK. Paediatr Perinat Drug Ther. 1997;2:52-55

11. Turner S, Gill A, Nunn T, Hewitt B, Choonara I. Use of "off-label" and unlicensed drugs in paediatric intensive care unit [letter]. Lancet. 1996; 347:549-550

12. Turner S, Longworth A, Nunn AJ, Choonara I. Unlicensed and off label drug use in paediatric wards: prospective study. BMJ. 1998;316:343-345

13. Conroy S, McIntyre J, Choonara I. Unlicensed and off label drug use in neonates. Arch Dis Child Fetal Neonatal Ed. 1999;80:F142-F144

14. McIntyre J, Conroy S, Avery A, Corns H, Choonara I. Unlicensed and off label prescribing of drugs in general practice. Arch Dis Child. 2000; 83:498-501

15. Chalumeau M, Treluyer JM, Salanave B, et al. Off label and unlicensed drug use among French office based paediatricians [in process citation]. Arch Dis Child. 2000;83:502-505

16. Gilman JT, Gal P. Pharmacokinetic and pharmacodynamic data collection in children and neonates. A quiet frontier [comment]. Clin Pharmacokinet. 1992;23:1-9

17. Guidance for Industry: Content and Format for Pediatric Use Supplements. Washington, DC: Food and Drug Administration, Center for Drug Evaluation and Research (CDER), Center for Biologics Evaluation and Research (CBER); 1996:1-6

18. Newman C, Hull D. Comment: regulations for pediatric labeling of prescription drugs [letter; comment]. Ann Pharmacother. 1997;31: 1092-1093

19. Department of Health and Human Services. Food and Drug Administration. 21 CFR Parts 201, 312, 314, and 601: Regulations Requiring Manufacturers to Assess the Safety and Effectiveness of New Drugs and Biological Products in Pediatric Patients. Rockville, MD: Food and Drug Administration, Center for Drug Evaluation and Research; 1998:66632-66672
20. Federal Register 21 CFR Part 201. Requirements on content and format of labeling for human prescription drugs and biologics. Requirements of prescription drug product labels; proposed rule; 2000

21. Kmietowicz Z. Drug industry is unwilling to run trials in children. BMJ. 2000;320:1362A

22. Impicciatore $\mathrm{P}, \mathrm{Choonara}$ I. Status of new medicines approved by the European Medicines Evaluation Agency regarding paediatric use. $\mathrm{Br} \mathrm{J}$ Clin Pharmacol. 1999;48:15-18

23. Dutch Society of Research-orientated Pharmaceutical Industry N, Dutch Society of the Phamaceutical Industry of Selfcare Medication and Healthproducts, Neprofarm. Repertorium 98/99, overview of by the Medication Evaluation Board registered information texts of pharmaceutical specialties. In: Repertorium. Utrecht, The Netherlands: Nefarma and Neprofarm; 1998

24. Central Medical Pharmaceutical Committee of the National Health Service Council. Farmaceutisch Kompas. Utrecht, The Netherlands: National Health Service Council; 1998

25. Conroy S, Choonara I, Impicciatore P, et al. Survey of unlicensed and off label drug use in paediatric wards in European countries. BMJ. 2000; 320:79-82

26. 't Jong GW, Vulto AG, de Hoog M, Schimmel KJ, Tibboel D, van den Anker JN. Unapproved and off-label use of drugs in a children's hospital [letter]. N Engl J Med. 2000;343:1125

27. The Pediatric Exclusivity Provision. January 2001 Status Report to Congress. Washington, DC: Department of Health and Human Services, US Food and Drug Administration; 2001

28. 't Jong GW, van den Anker JN, Choonara I. FDAMA's written request list: medicines for children [letter]. Lancet. 2001;357:398

29. Bonati M, Choonara I, Hoppu K, Pons G, Seyberth H. Closing the gap in drug therapy [letter]. Lancet. 1999;353:1625

\section{STUDY TO DEVELOP WAY TO MONITOR CANCER CARE}

Responding to a call to improve the quality of cancer care in the United States, a leading cancer organization said that it was working with other groups to develop a national system to monitor how well each cancer patient is treated.

... The ultimate goal is to develop specific measures of quality spanning the entire course of care, from diagnosis to cure or death. Such measures could then be used to hold health care providers accountable for the quality of cancer care.

... "There is no national cancer care program or system of care in the United States," the report said. It also said that the government had numerous cancer programs and research, "but in no one place are these disparate efforts coordinated or even described."

A substantial, but unmeasured, number of cancer patients do not receive care known to be effective for their condition, the medical institute said, and the reasons for failure to deliver high-quality care could come from overuse, underuse, or misuse of proper therapy but have not been studied adequately.

Conducting clinical trials to determine the safety and effectiveness of therapies is a first step in assessing quality. But too few patients participate in such trials even though studies show that routine care costs in cancer clinical trials are no more than in standard care, cancer experts have said. 


\section{A Survey of the Use of Off-Label and Unlicensed Drugs in a Dutch Children's Hospital}

Geert W. 't Jong, Arnold G. Vulto, Matthijs de Hoog, Kirsten J. M. Schimmel, Dick

Tibboel and John N. van den Anker

Pediatrics 2001;108;1089-1093

DOI: $10.1542 /$ peds.108.5.1089

This information is current as of November 20, 2006

$\begin{array}{ll}\begin{array}{l}\text { Updated Information } \\ \text { \& Services }\end{array} & \text { including high-resolution figures, can be found at: } \\ \text { http://www.pediatrics.org/cgi/content/full/108/5/1089 } \\ \text { References } & \text { This article cites } 16 \text { articles, } 8 \text { of which you can access for free } \\ & \text { at: } \\ & \text { http://www.pediatrics.org/cgi/content/full/108/5/1089\#BIBL } \\ & \text { This article has been cited by } 9 \text { HighWire-hosted articles: } \\ \text { http://www.pediatrics.org/cgi/content/full/108/5/1089\#otherarticl } \\ \text { es } \\ \text { This article, along with others on similar topics, appears in the } \\ \text { following collection(s): } \\ \text { Therapeutics \& Toxicology } \\ \text { http://www.pediatrics.org/cgi/collection/therapeutics_and_toxico } \\ \text { logy } \\ \text { Information about reproducing this article in parts (figures, } \\ \text { tables) or in its entirety can be found online at: } \\ \text { http://www.pediatrics.org/misc/Permissions.shtml } \\ \text { Permissions \& Licensing } & \text { Information about ordering reprints can be found online: } \\ & \text { http://www.pediatrics.org/misc/reprints.shtml }\end{array}$

